\title{
Heterogeneously Dense Breast
}

National Cancer Institute

\section{Source}

National Cancer Institute. Heterogeneously Dense Breast. NCI Thesaurus. Code C132103.

A mammographic finding of heterogeneously dense breast composition, as defined by the visually estimated content of fibroglandular-density tissue within the breast, based on updated editions of the American College of Radiology's Breast Imaging Reporting and Data System (BI-RADS). 